\title{
Central motor and sensory conduction in adrenoleukomyeloneuropathy, cerebrotendinous xanthomatosis, HTLV-1-associated myelopathy and tabes dorsalis
}

\author{
YOSHIKAZU UGAWA, NOBUO KOHARA, * TOMOYUKI SHIMPO, \\ TORU MANNEN
}

From the Department of Neurology, Institute of Brain Research, School of Medicine, University of Tokyo, and the Department of Neurology, ${ }^{*}$ Tokyo Metropolitan Geriatric Hospital, Tokyo, Japan

SUMMARY Central motor and sensory conduction was studied by percutaneous electrical stimulation of brain and spinal cord and by somatosensory evoked potential techniques respectively, in patients with adrenoleukomyeloneuropathy, cerebrotendinous xanthomatosis, human T-cell lymphotropic virus-1-associated myelopathy and tabes dorsalis. The results were all consistent with clinical and neuropathological findings in these disorders. Conductions in the corticospinal tract and posterior column could be evaluated separately with these two techniques. Percutaneous electrical stimulation technique would be useful for investigating conduction in the corticospinal tract in patients with spinal cord disorders.

The recently developed techniques of percutaneous electrical stimulation of the brain and spinal cord ${ }^{12}$ have allowed measurements to be made of central motor conduction in conscious human subjects. The normal values of central motor conduction times have been established, ${ }^{1-6}$ and delays have been demonstrated in multiple sclerosis, ${ }^{78}$ motor neuron disease $^{9-11}$ and cerebrovascular disease. ${ }^{11} 12 \mathrm{~A}$ few myelopathic disorders have been studied with this method, too. ${ }^{1314}$ In this study, we have measured conduction in both central motor pathways and central sensory pathways using this technique and conventional evoked potential techniques in patients with adrenoleukomyeloneuropathy (ALMN), cerebrotendinous xanthomatosis (CTX), human T-cell lymphotropic virus 1 (HTLV-1)-associated myelopathy (HAM) and tabes dorsalis. The electrophysiological results suggest differential involvement of central sensory and motor pathways in a manner compatible with clinical and pathological findings in these disorders.

Address for reprint requests: Dr Yoshikazu Ugawa, Dept of Neurology Institute of Brain Research, School of Medicine, University of Tokyo, Hongo 7-3-1, Bunkyo-ku, Tokyo 113, Japan.

Received 8 January 1988 and in revised form 15 March 1988. Accepted 2 April 1988

\section{Subjects}

Two patients with ALMN, four with CTX, four with HAM and one with tabes dorsalis were studied.

The two patients with ALMN had spastic paraparesis, mild deep sensory disturbances, difficulty in urination, decreased visual acuity and skin pigmentation. Patient 2 showed clinical signs of peripheral neuropathy, such as sensory disturbance of the glove-stocking type. Laboratory investigations disclosed adrenal insufficiency and elevation of very long chain fatty acid in both of them.

The four patients with CTX showed cerebellar symptoms, pyramidal tract signs, deep sensory disturbances, mental deterioration and characteristic swelling of the Achilles tendons. All of them had an increased cholestanol content in the blood and an excretion of abnormal bile alcohols in the urine.

The four patients with HAM suffered from spastic paraparesis. Their deep tendon reflexes of the lower extremities were hyperactive and plantar responses were extensor. They had mild deep sensory disturbances (patient 7-9) or no sensory disturbance (patient 10). We diagnosed all of these patients as HAM first described by Osame $e$ al $^{15}$ because of the highly positive anti-HTLV-1 antibody titres in both the serum and cerebrospinal fluid (CSF). All patients showed some improvement with predonisolone.

A 72-year-old man (patient 11) showed ataxic gait due to the disturbances of deep sensation and suffered from gastric crisis. He had severe deep sensory disturbances with Romberg's sign, while he had neither weakness nor 
Babinski's sign. The diagnosis (tabes dorsalis) was confirmed by the high titre of TPHA in serum and CSF; other causes of myelopathy were excluded by myelography and conventional means.

Patients with a history of convulsive seizures or cardiac disease were specifically excluded from the study.

\section{Methods}

Conduction in central sensory pathway was studied using somatosensory evoked potentials (SEPs). For the upper limb SEPs, the median nerve was stimulated at the wrist. Recordings were made from the ipsilateral Erb's point, the seventh and second cervical spinous process (CV7 and CV2, respectively) and the contralateral hand somatosensory area ( $7 \mathrm{~cm}$ lateral to the midline on a line drawn from a $2 \mathrm{~cm}$ posterior to the vertex to the external auditory meatus). A midfrontal $(\mathrm{Fz})$ electrode was used as reference. For the lower-limb SEPs, the posterior tibial nerve was stimulated at the ankle and lumbar potentials and cortical SEPs were monitored. Recordings were made from the first lumbar spinous process (L1) and referenced at the contralateral iliac crest. On the scalp, recordings were made from the foot somatosensory area ( $2 \mathrm{~cm}$ posterior to the vertex on the midline; $\mathbf{C z}$ ) and referenced to Fz. The stimulus strength was adjusted to a level which produced a minimal contraction of the abductor pollicis brevis or the abductor hallucis muscles. The potentials were amplified with filters set at $8 \mathrm{~Hz}$ and 3,000 $\mathrm{Hz}$, and at least 1,024 responses were averaged. The latencies of N9, N11, N13 and N20 components of the upper-limb SEPs and those of the lumbar potential (LP) and P37 component of the lower-limb SEPs were measured. The central sensory conduction times (CSCTs) were calculated as N13-N20 and LP-P37 interpeak times for the upper and lower limb SEPs, respectively. Normal values for all parameters have been established in 30 normal volunteers and the upper limits of the normal range (mean $+2.5 \mathrm{SD}$ ) are listed in the table.

Conduction in the central motor pathway was studied using percutaneous electrical stimulation of the brain and spinal cord, details of which have already been described. ${ }^{4-6}$ Motor action potentials of the limb muscles were recorded during cortical, cervical and lumbar stimulations by a specially constructed low-output impedance stimulator. To activate muscles in the arm, the anode was placed $7 \mathrm{~cm}$ laterally on a line from the vertex to the external auditory meatus and the cathode $5 \mathrm{~cm}$ anterior to the anode. For muscles in the leg, the anode was placed at the vertex and the cathode $5 \mathrm{~cm}$ anterior to the anode. For cervical stimulation, the anode was applied over the fifth cervical (C5) spinous process and the cathode over the $\mathrm{C} 7$ spinous process. For lumbar stimulation, the anode and the cathode were applied over the twelfth thoracic (T12) and the first lumbar (L1) spinous processes, respectively. In all recordings, the stimulation voltage was increased until a response of maximal amplitude was obtained. Patients were instructed to relax as fully as possible during the procedure. Recordings were made with surface electrodes in pairs about $5 \mathrm{~cm}$ apart over the muscle of interest. Compound muscle action potentials were recorded from the extensor carpi radialis (ECR) and thenar muscles in both arms and from the quadriceps femoris (Quad) and tibialis anterior (TA) muscles in both legs.
Recordings were also made from other muscles in some patients. Responses were amplified (bandpass $80 \mathrm{~Hz}-10$ $\mathrm{KHz}$ ) on an electroencephalograph which was interfaced with a microcomputer (Signal Processor 7T18, NEC San-Ei) for display, storage and plotting of responses. The time from the cortical stimulus to the onset of the evoked electromyographic (EMG) response in the relaxed muscle was taken as the cortical latent period (Lcor). The time from the cervical or lumbar stimulus to the onset of the EMG activity was taken as the spinal latent period (Lsp). The central motor conduction time (CMCT) was obtained by subtracting Lsp from Lcor for each muscle. The parameters measured in the relaxed condition were used, partly because some patients could not produce appropriate voluntary contraction and partly because the latencies were sometimes difficult to measure accurately with background muscle activity. Normal values for Lcor, Lsp and CMCT of the upper and lower limb muscles have already been established in 40 normal volunteers aged between 17 and 72 years. ${ }^{6}$ The upper limits of the normal range are listed in the table.

All patients gave their informed consent to the investigations. These procedures were approved by the Ethics Committee of Tokyo University Hospital.

\section{Results}

The results are summarised in the table. Since there were no differences between the right and left sides, the results of the right upper and lower limb SEPs and those of motor responses on the right side are summarised.

Central sensory conduction was considered abnormal if the CSCT was prolonged or cortical SEPs were absent despite a normal N13 component (median nerve SEP) or normal lumbar potential (posterior tibial nerve SEP). Central motor conduction was considered abnormal if the CMCT exceeded the upper limit of the normal range or if no motor response could be evoked by cortical stimulation despite a normal response to cervical or lumbar stimulations.

In two patients with ALMN, the CSCTs of median nerve SEPs were prolonged, although those of the N13 component were normal. The lumbar potential was normal in patient 1 and was absent in patient 2 , and the cortical components of posterior tibial nerve SEPs were absent in both of them. For both upper and lower limb muscles, the Lsps were normal in patient 1 and prolonged in patient 2, and CMCTs showed some abnormalities in both of them (fig 1). In patient 1 , the motor and sensory nerve conduction velocities were normal. In patient 2 , the right median nerve motor and sensory conduction velocities (MCV and SCV) between the elbow and the wrist were normal (MCV: $46.7 \mathrm{~m} / \mathrm{s}, \mathrm{SCV}: 69.4 \mathrm{~m} / \mathrm{s}$ ), while both MCV in the right peroneal nerve and SCV in the right sural nerve were reduced (MCV: $33.7 \mathrm{~m} / \mathrm{s}, \mathrm{SCV}: 38.9 \mathrm{~m} / \mathrm{s}$ ).

In all patients with CTX, the cervical component 
it Thenar

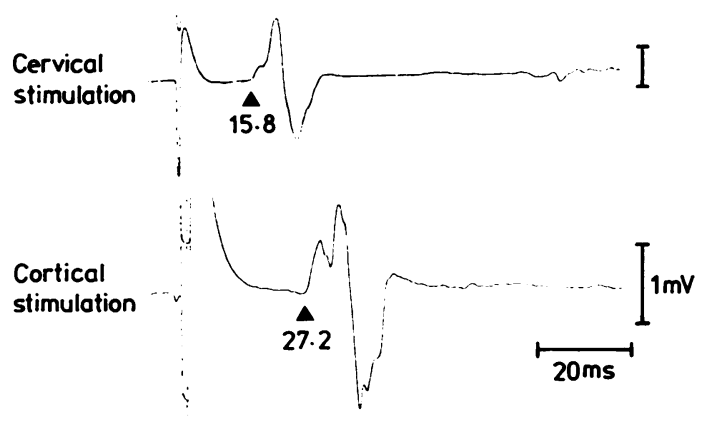

rt TA
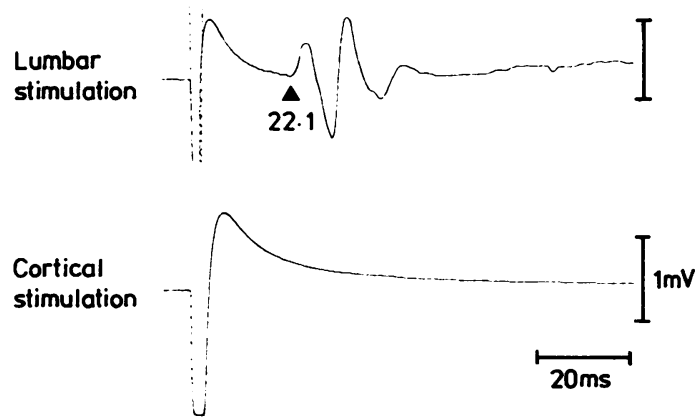

Fig 1 Central motor conduction to the right thenar and tibialis anterior (TA) muscles in case 2

(Adrenoleukomyeloneuropathy). Both the spinal latent period ( $L s p$ ) and central motor conduction time for the right thenar muscle were abnormally prolonged. The Lsp for the right TA muscle was $22.1 \mathrm{~ms}$ (the upper limit: $18.6 \mathrm{~ms}$ ), and no response could be evoked by cortical stimulation.

(N13) of the median nerve SEPs and lumbar potentials (N20) of the posterior tibial nerve SEPs were normal. The CSCTs of median nerve SEPs were prolonged in one out of three patients, and those of posterior tibial nerve SEP in all of two examined patients. Central motor conduction to the upper-limb muscles was abnormal in all three examined patients (in five out of seven muscles) and those to the lower-limb muscles in two examined patients (in all of six muscles), whereas the Lsps were normal in all muscles (table).

The CSCTs of the median nerve SEPs were within the normal range in all four patients with HAM, while those of the posterior tibial nerve SEPs were mildly delayed in one of four patients. The CMCTs to the upper limb muscles were all normal and central motor conduction to the lower-limb muscles was considered abnormal in all these patients. Figure 2 shows motor responses in the lower-limb muscles in one patient with HAM (case 10). There was no motor response to cortical stimulation in the Quad muscle and CMCTs to the TA and GC muscles were prolonged, although normal responses could be evoked by lumbar stimulation. The response of the FHB muscle to cortical stimulation lasted longer (temporal dispersion) than those in normal subject, despite its normal latency. The response in GC muscle was small and dispersed, too. In patients with HAM, therefore, central motor conduction was markedly involved although central sensory conduction was normal or only mildly involved. The results of SEP and central motor conduction studies after the treatment with predonisolone showed no significant differences from those before the treatment, even though the clinical signs were improved by treatment.

In a patient with tabes dorsalis, posterior tibial nerve SEPs were severely delayed at the cortex with no recordable lumbar potential. The median nerve SEP
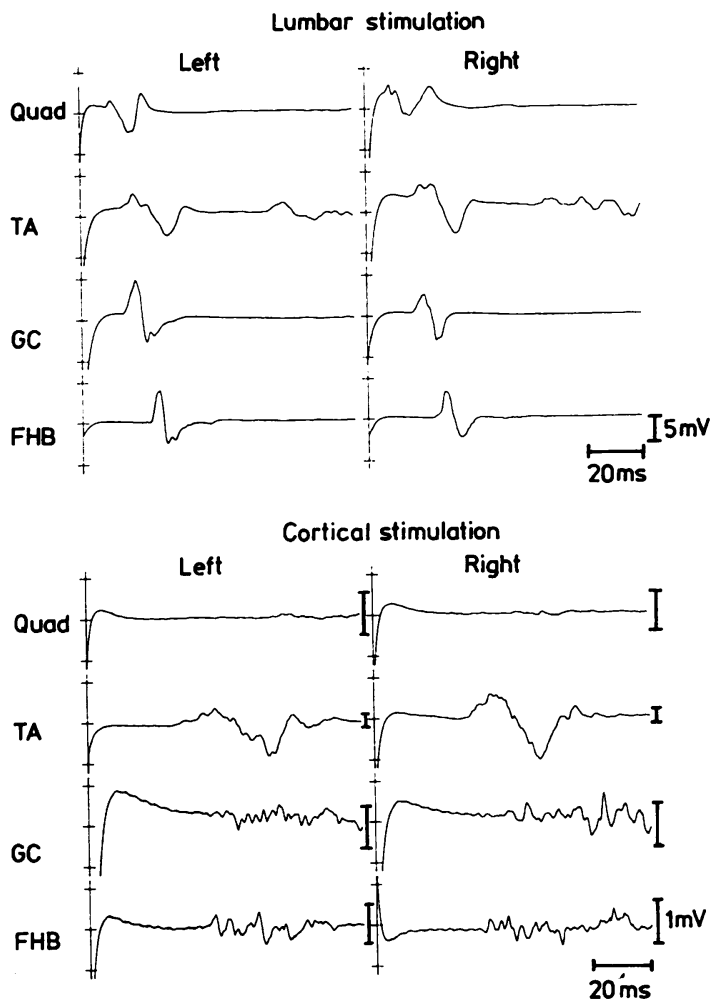

Fig 2 Motor responses in case 10 (HTLV-1-associated myelopathy). Motor responses to cortical stimulation in gastrocnemius and flexor hallucis brevis muscles were small and dispersed. No motor responses could be evoked by cortical stimulation in quadriceps femoris muscle. Quad: quadriceps femoris muscle; TA: tibialis anterior muscle; GC: gastrocnemius muscle; FHB: flexor hallucis brevis muscle. 
Table The electrophysiological results

\begin{tabular}{|c|c|c|c|c|c|c|c|c|c|}
\hline \multirow[b]{2}{*}{ Case } & \multirow[b]{2}{*}{ Diagnosis } & \multirow[b]{2}{*}{ Age (yr) } & \multirow[b]{2}{*}{ Sex } & \multicolumn{3}{|c|}{ Median Nerve } & \multicolumn{3}{|c|}{ Posterior Tibial Nerve } \\
\hline & & & & $N_{13}$ & $N_{20}$ & $\begin{array}{l}C S C T \\
\left(N_{13}-N_{20}\right)\end{array}$ & $\begin{array}{l}L P \\
\left(N_{20}\right)\end{array}$ & $P_{37}$ & $\begin{array}{l}C S C T \\
\left(N_{20}-P_{37}\right)\end{array}$ \\
\hline $\begin{array}{r}1 \\
2 \\
3 \\
4 \\
5 \\
6 \\
7 \\
8 \\
9 \\
10 \\
11\end{array}$ & $\begin{array}{l}\text { ALMN } \\
\text { ALMN } \\
\text { CTX } \\
\text { CTX } \\
\text { CTX } \\
\text { CTX } \\
\text { HAM } \\
\text { HAM } \\
\text { HAM } \\
\text { HAM } \\
\text { Tabes Dorsalis }\end{array}$ & $\begin{array}{l}33 \\
22 \\
26 \\
38 \\
43 \\
56 \\
45 \\
44 \\
61 \\
31 \\
72\end{array}$ & $\begin{array}{l}\mathbf{M} \\
\mathbf{M} \\
\mathbf{F} \\
\mathbf{F} \\
\mathbf{M} \\
\mathbf{M} \\
\mathbf{M} \\
\mathbf{F} \\
\mathbf{F} \\
\mathbf{F} \\
\mathbf{M}\end{array}$ & $\begin{array}{l}13 \cdot 0 \\
13 \cdot 0 \\
14 \cdot 0 \\
12 \cdot 2 \\
13 \cdot 2 \\
13 \cdot 3 \\
13 \cdot 6 \\
13 \cdot 7 \\
12 \cdot 3 \\
14 \cdot 4\end{array}$ & $\begin{array}{l}21 \cdot 4 \\
22 \cdot 3 \\
22 \cdot 4 \\
18 \cdot 2 \\
19 \cdot 8 \\
* \\
19 \cdot 3 \\
19 \cdot 6 \\
20 \cdot 3 \\
18 \cdot 7 \\
20.6\end{array}$ & $\begin{array}{l}8 \cdot 4 \\
9 \cdot 3 \\
8 \cdot 4 \\
6 \cdot 0 \\
6 \cdot 6 \\
* \\
6 \cdot 0 \\
6 \cdot 0 \\
6 \cdot 6 \\
6 \cdot 4 \\
6 \cdot 2\end{array}$ & $\begin{array}{l}22 \cdot 1 \\
\text { NR } \\
* \\
18 \cdot 2 \\
21 \cdot 8 \\
* \\
17 \cdot 5 \\
21 \cdot 2 \\
20 \cdot 7 \\
19 \cdot 5 \\
\text { NR }\end{array}$ & $\begin{array}{l}\text { NR } \\
\text { NR } \\
* \\
43 \cdot 6 \\
47 \cdot 9 \\
* \\
36 \cdot 7 \\
44 \cdot 0 \\
40 \cdot 7 \\
40 \cdot 0 \\
54 \cdot 2\end{array}$ & $\begin{array}{l}* \\
25.4 \\
26.1 \\
* \\
19.2 \\
22.8 \\
20.0 \\
20.5\end{array}$ \\
\hline $\begin{array}{c}\text { Contro } \\
\text { Mea } \\
\text { SD } \\
\text { Mea }\end{array}$ & $2.5 \mathrm{SD}$ & & & $\begin{array}{r}13.5 \\
0.7 \\
15.3\end{array}$ & $\begin{array}{r}19 \cdot 1 \\
0.8 \\
21 \cdot 1\end{array}$ & $\begin{array}{l}5 \cdot 5 \\
0 \cdot 5 \\
6 \cdot 8\end{array}$ & $\begin{array}{r}21.4 \\
1.2 \\
24.4\end{array}$ & $\begin{array}{r}38.8 \\
1.4 \\
42.3\end{array}$ & $\begin{array}{r}17.5 \\
1.2 \\
20.5\end{array}$ \\
\hline
\end{tabular}

ALMN: Adrenoleukomyeloneuropathy, CTX: Cerebrotendinous Xanthomatosis.

HAM: Human T-cell lymphotropic virus 1 (HTLV-1)-associated myelopathy.

CSCT: Central sensory conduction time, CMCT: Central motor conduction time.

Del: Deltoid, Bi: Biceps brachii, ECR: Extensor carpi radialis.

TA: Tibialis anterior, GC: Gastrocnemius, FHB: Flexor hallucis brevis.

NR: no response, ": not done.

was normal (fig 3). The CMCTs to all upper and lower limb muscles were normal. The motor conduction velocity was $48.9 \mathrm{~m} / \mathrm{s}$ in the left peroneal nerve and the sensory conduction velocity of the left sural nerve was $44.4 \mathrm{~m} / \mathrm{s}$. The amplitude of the sensory nerve action
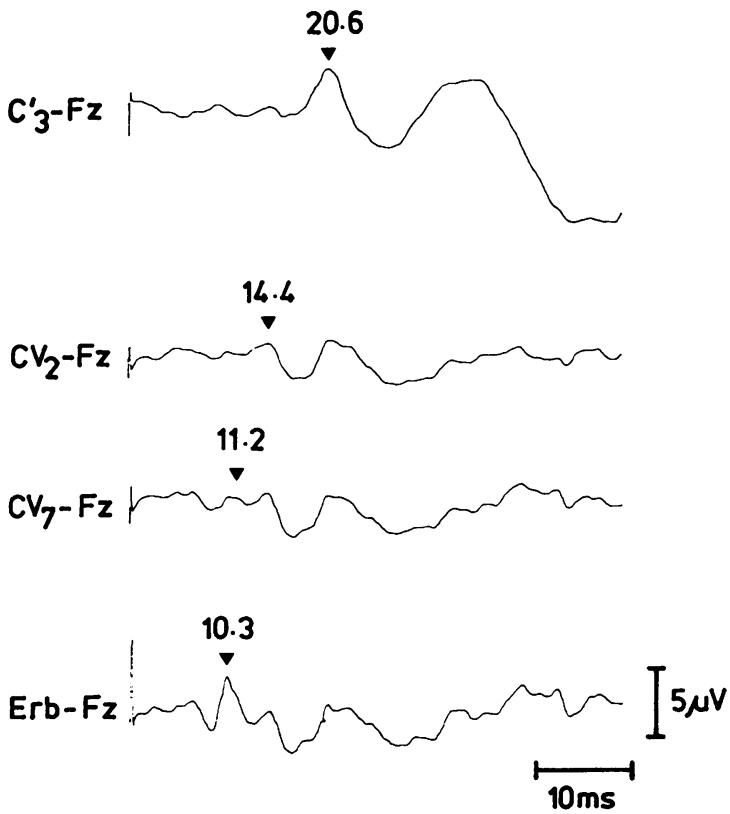

potential was $3.7 \mu \mathrm{V}$ in the left sural nerve. Peripheral motor and sensory conduction velocities were normal in all other nerves examined in this patient.

No untoward side effects of cortical stimulation were noted in any of the patients.
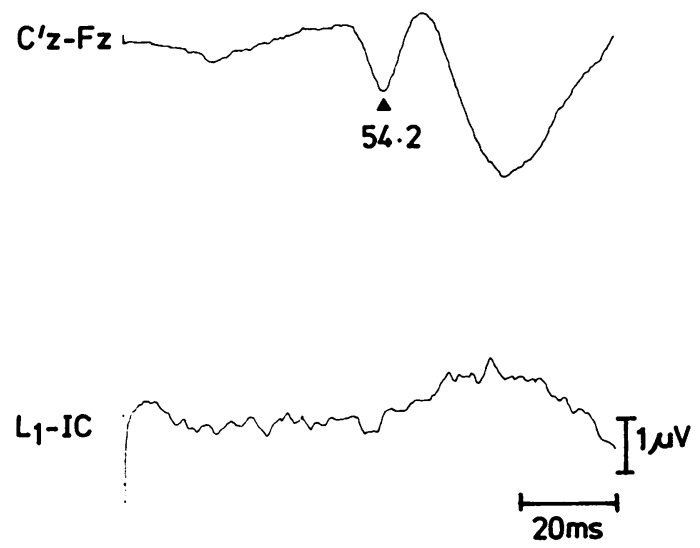

Fig 3 SEPs in case 11 (tabes dorsalis). The lumbar potential was absent and the latency of P37 component was $54.2 \mathrm{~ms}$ (upper limit: $42 \cdot 3 \mathrm{~ms}$ ), while median nerve SEP was normal. C3': left hand somatosensory area; Fz: mid-frontal; CV2, CV7: second, and seventh cervical vertebrae, respectively; $C_{z}$ ': leg somatosensory area; L1: first lumbar spinous process; IC: iliac crest. 


\begin{tabular}{|c|c|c|c|c|c|c|c|}
\hline \multicolumn{8}{|c|}{$C M C T$} \\
\hline Del & $B i$ & $E C R$ & Thenar & Quad & $T A$ & $G C$ & $F H B$ \\
\hline$*$ & $*$ & 15.9 & 15.7 & NR & NR & $*$ & $*$ \\
\hline$*$ & $*$ & $11 \cdot 1$ & 11.4 & NR & NR & * & * \\
\hline$*$ & $*$ & $*$ & $*$ & $*$ & & $*$ & $*$ \\
\hline$*$ & $*$ & $*$ & $14 \cdot 0$ & $*$ & $*$ & $*$ & $*$ \\
\hline$*$ & $*$ & 16.4 & 12.5 & NR & NR & $*$ & $*$ \\
\hline NR & $7 \cdot 3$ & 9.8 & $11 \cdot 9$ & NR & NR & NR & $20 \cdot 1$ \\
\hline * & $*$ & 6.8 & 5.8 & NR & NR & NR & $19 \cdot 0$ \\
\hline$*$ & $*$ & 6.9 & $9 \cdot 1$ & NR & $22 \cdot 5$ & NR & $17 \cdot 2$ \\
\hline $7 \cdot 2$ & $6 \cdot 8$ & $7 \cdot 5$ & $8 \cdot 5$ & NR & NR & NR & $19 . \overline{9}$ \\
\hline$*$ & $*$ & $6 \cdot 1$ & 8.8 & NR & $17 \cdot 3$ & $28 \cdot 2$ & $16 \cdot 4$ \\
\hline $5 \cdot 8$ & $5 \cdot 4$ & $6 \cdot 7$ & 6.6 & $12 \cdot 2$ & $13 \cdot 1$ & 13.0 & 13.6 \\
\hline 6.5 & 6.8 & $7 \cdot 3$ & $7 \cdot 6$ & $12 \cdot 9$ & $13 \cdot 2$ & 13.0 & 14.4 \\
\hline 0.5 & 0.6 & $1 \cdot 2$ & $1 \cdot 2$ & 1.4 & $1 \cdot 6$ & $1 \cdot 3$ & 1.9 \\
\hline $7 \cdot 7$ & $8 \cdot 3$ & $10 \cdot \overline{3}$ & 10.6 & 16.4 & $17 \cdot 2$ & $16 \cdot 3$ & $19 \cdot 2$ \\
\hline
\end{tabular}

\section{Discussion}

Results in control subjects, ${ }^{4513}$ in patients with cerebrovascular $^{1112}$ or motor neuron disease ${ }^{9-12}$ suggest that electrical stimulation of brain and spinal cord is a test of conduction in the corticospinal tract. We used this technique together with conventional SEP testing of dorsal column function ${ }^{16}$ to study central motor and sensory conduction in four disorders.

The results are compared with the clinical features and neuropathological findings in each disorder.

Both central motor and sensory conduction were abnormal in both patients with ALMN. These results were consistent with the neuropathological findings of ALMN. Neuropathological studies demonstrated diffuse involvement of the white matter in the brain and spinal cord; both the tract of deep sensation and the corticospinal tract were damaged. The absence of lumbar sensory potential and prolongation of the Lsps in patient 2 were probably due to the involvement of peripheral nerves. Prolongation of conduction time along a short segment of motor roots may cause an increase in CMCT in some patients with neuropathy ${ }^{17}$ However, judging from the motor conduction velocities of the peripheral nerves and the degrees of prolongation of Lsp or CMCT, we concluded that the conduction in the corticospinal tract was abnormal in this patient.

In patients with CTX, peripheral conduction was normal although both central motor and sensory conductions frequently were involved. The results were consistent with clinical findings and also with neuropathological observation that there is demyelination of both the corticospinal tract and posterior column. ${ }^{18}$ The fact that some abnormalities of central motor and sensory conduction were more frequent in the lower-limbs than in the upper-limbs also was consistent with the neuropathological findings of a distal axonal degeneration. ${ }^{18}$

In one of four patients with HAM, the CSCT of the posterior tibial nerve SEP was longer than normal. In all of them, central motor conduction to the lowerlimb muscles was abnormal, although that to the upper-limb muscles was normal. The motor roots might be mildly involved in some patients with HAM. ${ }^{19}$ Since these mild involvements would not cause the severe abnormalities in the central motor conduction demonstrated in our patients, our results suggested that the corticospinal tract was involved in the patients with HAM. This disease was thought to be a kind of myelopathy with prominent corticospinal tract signs and mild sensory disturbance. ${ }^{15}$ The electrophysiological results were consistent with these clinical features in this disorder. The fact that central motor conduction was abnormal only in the leg is further evidence that the spinal cord is involved in this disease.

In a patient with tabes dorsalis, the posterior tibial nerve SEP was markedly abnormal, whereas central motor conduction was normal in all of the upper and lower limb muscles. These results were consistent with the neuropathological observations that the corticospinal tract was intact despite severely degenerated posterior columns in tabes dorsalis. ${ }^{20}$ The result that the median nerve SEP was normal in spite of abnormal posterior tibial nerve SEP was compatible with the clinical feature that he had more severe deep sensory disturbances in the lower extremities than in the upper extremities and also consistent with the neuropathological findings that the posterior columns from the cervical segments were more mildly degenerated than those from the lumbar segments. In this 
patient, the lumbar potential could not be recorded although both the sensory conduction velocity and the amplitude of the sensory nerve action potential were normal in the sural nerve. The absence of lumbar potential was also consistent with the neuropathological findings that the dorsal roots were severely degenerated as well as the dorsal columns in tabes dorsalis, ${ }^{20-22}$ because the lumbar potential was thought to be generated in the root entry zone of the spinal cord. ${ }^{16}$

The technique of percutaneous electrical stimulation of central motor pathway has been applied clinically to a few kinds of spinal cord diseases, such as radiation myelopathy ${ }^{13}$ and cervical spondylosis. ${ }^{14}$ In this report, we confirm the usefulness of this technique in the diagnosis of patients with various spinal cord diseases. We stress here that the lower-limb muscles have to be studied in patients with spinal cord diseases, as in motor neuron disease. ${ }^{11}$

We are very grateful to $M$ Nishizawa, MD and $T$ Ohno, MD for their measurement of very long chain fatty acid, K Kasama, MD for measurement of cholestanol and M Hayami, MD for measurement of anti-HTLV-1 antibody titer. We are also very grateful to Dr J C Rothwell (Institute of Psychiatry, London) for helpful suggestions and criticism on the manuscript. We thank Miss Kieko Genba for her expert technical assistance.

This work was supported in part by Grant No. 86-15 from National Centre of Neurology and Psychiatry (NCNP) of the Ministry of Health and Welfare, Japan.

\section{References}

1 Merton PA, Morton HB. Stimulation of the cerebral cortex in the intact human subject. Nature 1980;285:227.

2 Merton PA, Hill DK, Morton HB, Marsden CD. Scope of technique for electrical stimulation of human brain, spinal cord, and muscle. Lancet 1982;ii:597-600.

3 Rossini PM, DiStefano E, Stanzione P. Nerve impulse propagation along central and peripheral fast conducting motor and sensory pathways in man. Electroencephalogr Clin Neurophysiol 1985;60:320-34.

4 Ugawa Y, Kohara N, Kunimoto M, Mannen T. Physiologic analysis of central motor pathways. Clin Neurol (Tokyo) $1987 ; 27: 434-41$.
5 Ugawa Y, Kohara N, Kunimoto M, Mannen T. Physiologic analysis of central motor pathways. Abstract. Electroencephalogr Clin Neurophysiol 1987;66:95.

6 Genba K, Ugawa Y, Shimpo T, Mannen T. Functional analysis of central motor pathways. Neurol Med (Jap) 1987;27:581-4.

7 Cowan JMA, Rothwell JC, Dick JPR, Thompson PD, Day BL, Marsden CD. Abnormalities in central motor pathway conduction in multiple sclerosis. Lancet 1984;ii:304-7.

8 Mills KR, Murray NMF. Corticospinal tract conduction time in multiple sclerosis. Ann Neurol 1985;18:601-5.

9 Ingram DA, Swash M. Central motor conduction is abnormal in motor neuron disease. J Neurol Neurosurg Psychiatry 1987;50:159-66.

10 Berardelli A, Inghilleri M, Formisano R, Accornero N. Stimulation of motor tracts in motor neuron disease. J Neurol Neurosurg Psychiatry 1987;50:732-7.

11 Ugawa Y, Skimpo T, Mannen T. Central motor conduction in motor neuron disease and cerebrovascular disease. Acta Neurol (in press).

12 Thompson PD, Day BL, Rothwell JC, Dick JPR, Cowan JMA, Asselman P, Griffin GB, Sheehy MP, Marsden CD. The interpretation of electromyographic responses to electrical stimulation of the motor cortex in diseases of the upper motor neurone. J Neurol Sci 1987;80:91-110.

13 Snooks SJ, Swash M. Motor conduction velocity in the human spinal cord: slowed conduction in multiple sclerosis and radiation myelopathy. $J$ Neurol Neurosurg Psychiatry 1985;48:1135-9.

14 Thompson PD, Dick JPR, Asselman P, et al. Examination of motor function in lesions of the spinal cord by stimulation of the motor cortex. Ann Neurol 1987;21:389-96.

15 Osame M, Matsumoto M, Usuku K et al. Chronic progressive myelopathy associated with elevated antibodies to human $\mathrm{T}$ lymphotropic virus type I and adult T-cell leukemialike cells. Ann Neurol 1987;21:117-22.

16 Chiappa KH, Yiannikas C. Short latency somatosensory evoked potentials: interpretation. In: Chiappa KH, ed. Evoked Potentials in Clinical Medicine. New York: Raven Press, 1983:251-312.

17 Aizawa H, Ugawa Y, Shimpo T, Mannen T, Genba K. Conduction in the central and peripheral nervous system in neuropathies. Abstract. Jap J Electroencephalogr Electromyogr 1987; 15:133.

18 Pop PHM, Joosten E, van Spreeken A, et al. Neuroaxonal pathology of central and peripheral nervous system in cerebrotendinous xanthomatosis (CTX). Acta Neuropathol (Berl) 1984;64:259-264.

19 Arimura K, Rosales R, Osame M, Igata A. Clinical electrophysiologic studies of HTLV-1-associated myelopathy. Arch Neurol 1987;44:609-12.

20 Harriman DGF. Bacterial infection of the central nervous system. In: Adams JH, Corsellis JAN, Duchen LW, eds. Greenfields's Neuropathology. London: Edward Arnold, 1984:236-59.

21 Kunimoto M, Sakuta M, Takemura T, Inoue K, Nagahashi K. Dorsal root ganglion cells are well preserved in tabes dorsalis. Abstract. Neuropathol (Tokyo) 1984;5:307.

22 Lambert EH, Dyck PJ. Compound action potentials of sural nerve in vitro in peripheral neuropathy. In: Dyck PJ, Thomas PK, Lambert EH, Bunge R, eds. Peripheral Neuropathy. Philadelphia: Saunders, 1984:1030-44. 